\title{
Reliability, comparative validity and stability of dietary patterns derived from an FFQ in the Tehran Lipid and Glucose Study
}

\author{
Golaleh Asghari ${ }^{1}$, Arezoo Rezazadeh ${ }^{1}$, Firoozeh Hosseini-Esfahani ${ }^{1}$, Yadollah Mehrabi $^{2}$, \\ Parvin Mirmiran $^{1,3 *}$ and Fereidoun Azizi ${ }^{4}$ \\ ${ }^{1}$ Obesity Research Center, Research Institute for Endocrine Sciences, Shabid Beheshti University of Medical Sciences, Tehran, \\ Islamic Republic of Iran \\ ${ }^{2}$ School of Public Health, Shahid Beheshti University of Medical Sciences, Tehran, Islamic Republic of Iran \\ ${ }^{3}$ Department of Clinical Nutrition and Dietetics, Faculty of Nutrition Sciences and Food Technology, National Nutrition and \\ Food Technology Research Institute, Shahid Beheshti University of Medical Sciences, PO Box 19395-4741, Tehran, Islamic \\ Republic of Iran \\ ${ }^{4}$ Endocrine Research Center, Research Institute for Endocrine Sciences, Shabid Beheshti University of Medical Sciences, \\ Tehran, Islamic Republic of Iran
}

(Submitted 15 March 2011 - Final revision received 13 October 2011 - Accepted 22 October 2011 - First published online 17 January 2012)

\begin{abstract}
The aim of the present study was to assess the reliability, comparative validity and stability of dietary patterns defined by factor analysis for participants of the Tehran Lipid and Glucose Study. A total of 132 subjects, aged $\geq 20$ years, completed a 168-item FFQ (FFQ1, FFQ2) twice, with a 14-month interval. Over this duration, twelve dietary recalls (DR) were collected each month. To assess the stability of the FFQ, participants completed the third FFQ (FFQ3) after 8 years. Following these, two dietary patterns - the 'Iranian Traditional' and the 'Western' - were derived from FFQ1 and FFQ2 and the mean of DR (mDR); and three dietary patterns were identified from FFQ3: the 'Iranian Traditional', the 'Western' and the 'Combined'. The reliability correlations between factor scores of the two FFQ were 0.72 for the Iranian Traditional and 0.80 for the Western pattern; corrected month-to-month variations of DR correlations between the FFQ2 and mDR were 0.48 for the first and 0.75 for the second pattern. The $95 \%$ limits of agreement for the difference between factor scores obtained from FFQ2 and mDR lay between -1.58 and +1.58 for the Iranian Traditional and between -1.33 and +1.33 for the Western pattern. The intra-class correlations between FFQ2 and FFQ3 were $-0.09(P=0.653)$ and $0.49(P<0.001)$ for the 'Iranian Traditional' and the 'Western', respectively. These data indicate reasonable reliability and validity of the dietary patterns defined by factor analysis. Although the Western pattern was found to be fairly stable, the Iranian Traditional pattern was mostly unstable over the 8 years of the study period.
\end{abstract}

\section{Key words: Dietary patterns: Factor analysis: Reliability: Comparative validity: Stability}

Most previous literature in the field of nutrition discusses the relationship between disease and single nutrients or foods. According to $\mathrm{Hu}$ et al. ${ }^{(1)}$, there are high correlations between nutrients and foods, and there is a possibility of synergistic actions when these are consumed together in a meal. Consumption of a single food is commonly associated with a certain individual food pattern. Hence, in order to overcome these limitations, Hu et al. ${ }^{(2)}$ suggested that analysis of dietary patterns is a useful method to obtain real dietary combinations. Furthermore, dietary pattern analysis is applied in order to obtain a better perception of eating behaviours of different population groups and to design and implement effective dietary interventions for targeted groups $^{(3)}$. Dietary patterns, defined by factor analysis, models interrelated variables (food groups) as appearances of combinatory factors ${ }^{(4)}$. Togo et al. ${ }^{(4)}$ stated 'The factors are weighted combinations of foods, which best explain the variance in the food intake (the correlation matrix)'. Factor scores reflect the value of each of the foods that identifies the 'Factors', (4) . The FFQ or dietary recall is a common dietary assessment questionnaire used to define dietary patterns ${ }^{(5)}$. Using data collected by an FFQ, representative of the long-term diet, is more preferable than short-term methods like dietary recall to study dietary behaviours ${ }^{(6)}$.

Abbreviations: DR, dietary recalls; FCT, food composition table; mDR, mean of dietary recalls; TLGS, Tehran Lipid and Glucose Study; USDA, United States Department of Agriculture.

*Corresponding author: Professor P. Mirmiran, fax +98 21 220360657, email mirmiran@endocrine.ac.ir 
The reliability and comparative validity of FFQ represent the consistency of data at different time points when patterns may have not changed' and 'the ability of an FFQ to discriminate between subjects with true dietary intake differences', respectively ${ }^{(7)}$. Hence, the reliability and comparative validity of dietary patterns use FFQ data to show precision and accuracy of studied patterns. Stability shows the constancy of dietary intakes when it may have changed ${ }^{(8)}$. When the same dietary pattern is seen in a person over years, it is a stable dietary characteristic, and is important because many of the dietary parameters (individual foods and nutrients) seem to vary largely over time; furthermore, most studies on reliability and validity of dietary patterns have been conducted in western countries and assessed by principal component analysis ${ }^{(1,4,9,10)}$; however, to our knowledge, in Asian countries with different cultures and dietary habits, only one similar study has been performed in a Japanese population while no similar study has been conducted in the Middle East region. The FFQ used for the Iranian population in the Tehran Lipid and Glucose Study (TLGS) was validated for nutrients $^{(11)}$ and food group intakes ${ }^{(12)}$ but not for dietary patterns. The aim of the present study hence was to assess the reliability and comparative validity of dietary patterns defined by factor analysis, using dietary data collected with FFQ and $24 \mathrm{~h}$ dietary recalls (DR), and to assess the stability of dietary patterns after 8 years of follow-up of participants of the TLGS.

\section{Experimental methods}

The present study was initiated in 2002 and conducted on a subsample of TLGS subjects, urban residents of district-13 of Tehran, the capital of the Islamic Republic of Iran. Of the 15005 participants of the TLGS (1999-2001) ${ }^{(13)}$, a random sample of 200 cohort members, aged 20 to 70 years, was invited to participate in the present study; of the 200 enrolled, 162 agreed (participation rate: 81\%) and they were proportionately distributed across five 10-year age groups and two sexes to facilitate generalisation of the results to all age groups and both sexes. Sample size was determined by considering a CI of $95 \%$ and study power of $80 \%$, a minimum expected correlation coefficient of 0.25 and attrition rate estimation of $50 \%$. The inclusion criteria for the present study were current residence in Tehran for over 3 years and no history of diabetes or renal or liver disease. We excluded participants who did not satisfactorily complete the FFQ ( $n$ 12), had more than two missing $24 \mathrm{~h}$ DR ( $n$ 15), or those who had received a diagnosis of a chronic disease during the study period ( $n$ 3); a total of 132 subjects (sixty-one males and seventy-one females) remained for the analysis of reliability and validity. To assess stability of the dietary patterns, these individuals were followed-up for 8 years (2011). A total of fourteen subjects refused to complete the FFQ and twenty-nine subjects migrated and we did not have access to them; hence, eighty-nine subjects completed the FFQ. This study was conducted according to the guidelines laid down in the Declaration of Helsinki and all procedures involving human subjects were approved by the ethics committee of the Research Institute for Endocrine Sciences of the Shahid
Beheshti University of Medical Sciences. Written informed consent was obtained from all subjects.

\section{Assessment of dietary intake}

During the first phase of study, twelve $24 \mathrm{~h}$ DR were collected from each subject monthly. The first FFQ was completed 1 month before collection of the first $24 \mathrm{~h}$ DR (FFQ1), the second, 1 month after the last $24 \mathrm{~h} \mathrm{DR}$ (FFQ2) and the third at the end of follow-up (FFQ3). Data were collected by trained dietitians, with at least 3-5 years of experience in the nationwide food consumption survey and the TLGS ${ }^{(14,15)}$ and for each participant, completion of both questionnaires was supervised by the same dietitian.

The FFQ, originally designed for the TLGS, a semi-quantitative 168-item FFQ used to obtain information on dietary intake over a 1-year period, was a Willett format questionnaire ${ }^{(16)}$ modified based on Iranian food items. It included a list of foods (with standard serving sizes) commonly consumed by Iranians ${ }^{(14)}$. Individuals were requested to report their frequency of consumption of a given serving of each food item during the past year, on a daily, weekly, monthly or yearly basis. A standard portion size was designated for each item by using United States Department of Agriculture (USDA) serving sizes (e.g. bread, one slice; dairy, one cup); however, household measures were used for some items that were difficult to report by USDA standards (e.g. beans, one tablespoon; chicken meat, one leg, breast or wing; rice, one large, medium or small plate). The frequency reported for each food item was then converted to a daily intake. Portion sizes of consumed foods were converted to grams by using household measures ${ }^{(17)}$. The weight of seasonal items, such as some vegetables, was calculated based on the number of seasons that each food was available. Furthermore, twelve $24 \mathrm{~h}$ DR were collected, which included the two formal weekend days (Thursday and Friday in Iran) and ten weekdays for each subject. All recall interviews were performed at subjects' homes to estimate more precisely commonly used household measures and to limit the number of missing subjects. Researchers checked and resolved any vague recalls. Mixed foods in the $24 \mathrm{~h}$ DR were later converted into their ingredients. Since a very limited number of raw food items and nutrients can be analysed by the Iranian food composition table $(\mathrm{FCT})^{(18)}$, the USDA FCT ${ }^{(19)}$ was used as the main FCT to compute energy and nutrient intakes of food items; the Iranian FCT was used as an alternative for traditional Iranian food items, like 'kashk', which are not included in the USDA FCT.

\section{Food grouping}

Food items included in the FFQ and DR were grouped in certain food groups based on the resemblance of nutrients in foods ${ }^{(1)}$. Also, food grouping from other studies was considered a schema ${ }^{(1,20,21)}$. Iranians eat somewhat differently from people of other cultures. Taking this a priori knowledge of diet in Iran into account, plus knowledge of which foods are eaten and how they are prepared, our food groupings were altered. Some food items were considered in a separated 
group because their nutrient content was unique (e.g. eggs); thus, for both the $24 \mathrm{~h} \mathrm{DR}$ and FFQ data, food items were allocated to nineteen food groups (Table 1) and the amounts of each item in grams were summed to obtain the daily intake of each food group.

\section{Statistical analysis}

Data were analysed using SPSS 16 (SPSS, Inc.). The Kolmogorov-Smirnov test and histograms were used to test the normality of variables. Means of each food group, energy and nutrient intakes of twelve $24 \mathrm{~h}$ DR were calculated. Food group intakes were energy-adjusted by the residual $\operatorname{method}^{(22)}$. Significant differences in energy-adjusted food group intakes between the two dietary assessment methods, i.e. the $24 \mathrm{~h}$ DR and FFQ, were analysed by the Wilcoxon test, and the correlations between them were analysed by the Spearman correlation test. Factor analysis was used to identify major dietary patterns based on the nineteen food groups; factor analysis was performed separately for each FFQ and the mean of twelve $24 \mathrm{~h}$ DR food groups. The derived food groups at baseline were applied to FFQ3 to extract factor scores. Of the derived factors, the interpretable factors were retained based on Scree plots ${ }^{(23)}$, when an orthogonal rotation method, the Varimax rotation, was used to obtain explainable factors. The factors derived were labelled on the basis of interpretation of the data obtained in the present study and the results available in the literature. The factor score for each pattern and for each individual was computed by summing the standardised intakes of each food groups, weighted by the factor loadings ${ }^{(23)}$, and following this, each individual received a factor score for each identified pattern.
To examine the reliability, intra-class correlation was used between similar dietary pattern scores derived from the dietary data of FFQ1 and FFQ2. To examine the stability of dietary patterns, intra-class correlation was used between FFQ2 and FFQ3. Spearman correlation coefficients were calculated for evaluating the correlation between dietary pattern scores derived from FFQ2 and the mean of DR (mDR), to achieve comparative validity of dietary patterns derived from FFQ. In this study, the correlation between FFQ2 and DR was used for assessing validity, because FFQ2 represents the time period during which the $24 \mathrm{~h}$ DR were collected ${ }^{(12,24)}$. Correlations between food groups from the mDR and those from FFQ2 might, however, be higher than correlations for food groups from FFQ1. This difference indicates considerable learning bias and change of dietary intake over the year ${ }^{(24,25)}$

To reduce the random within-person, month-to-month variation in food pattern scores derived from the twelve DR, factor analyses were carried out on the food group intakes of each twelve $24 \mathrm{~h}$ DR and the deattenuated correlation coefficients for each dietary pattern score were calculated using the Rosner and Willett formula ${ }^{(26)}$.

Furthermore, Spearman correlation coefficients were used to evaluate the association between each dietary pattern score and the energy-adjusted mean of nutrients derived from the twelve $24 \mathrm{~h}$ DR. The agreement obtained between factor scores derived from FFQ2 and mDR was analysed by calculating the intra-class correlation coefficients and using the Bland and Altman method ${ }^{(27)}$, which depicted a plot of the difference between FFQ2 and DR against the mean of FFQ2 and MDR to determine how much the factor scores derived from FFQ2 differ from those derived by the DR as a 'gold standard' method. If there is no great difference between

Table 1. Food groupings used in the dietary pattern analysis

\begin{tabular}{|c|c|}
\hline Food groups & Food items \\
\hline Red meats & Beef, lamb, calf, chopped meat, hamburger \\
\hline Organ meats & Liver, kidney, heart, brain \\
\hline Poultry, fish and other seafood & Chicken, canned tuna fish, every kind of fish, shrimp \\
\hline Fast foods & Fried potatoes, French fries, sausages, pizza \\
\hline Eggs & Eggs \\
\hline Carbonated drinks & Coca-cola, other carbonated beverages, low-energy carbonated beverages \\
\hline Dairy products & $\begin{array}{l}\text { Low- and high-fat milk, yoghurt and cheese, yoghurt drink, chocolate milk, concentrated and creamy yoghurt, } \\
\text { ice cream, cream cheese, other cheeses, kashk }\end{array}$ \\
\hline Fruits and dried fruits & $\begin{array}{l}\text { Orange, tangerine, lemon, lime, grapefruit, banana, apple, pear, strawberry and other berries, peach, cherries, } \\
\text { fig, melon, watermelon and persian melon, cantaloupe, raisins or grapes, kiwi, apricots, nectarine, mulberry, } \\
\text { plums, persimmons, pomegranates, date, tinned fruits, natural fruit juices, other dried fruits }\end{array}$ \\
\hline Vegetables & $\begin{array}{l}\text { Cabbage, cauliflower, Brussels sprouts, kale, carrots, tomatoes, tomato sauce, tomato pasta, spinach, lettuce, } \\
\text { cucumber, eggplant, celery, green peas, green beans, green pepper, turnip, maize, squash, zucchini, } \\
\text { mushrooms, onions and garlic, green leafy vegetables }\end{array}$ \\
\hline Legumes and nuts & $\begin{array}{l}\text { Beans, peas, lima beans, broad beans, lentils, soya, peanuts, almonds, pistachios, walnuts, hazelnuts, } \\
\text { roasted seeds }\end{array}$ \\
\hline Potatoes & Potatoes \\
\hline Whole grains & Iranian dark breads, barley bread, barley, bulgur \\
\hline Refined grains & White breads (lavash, baguettes), noodles, pasta, rice, toasted bread, sweet bread, white flour, biscuits \\
\hline Salty snacks and vegetables & Potato chips, corn puffs, crackers, biscuits, popcorn, pickled vegetables \\
\hline Animal fats & Animal fats, butter, cream \\
\hline Vegetable oils & Vegetable oils, hydrogenated vegetable oils, mayonnaise, margarine \\
\hline Olives & Olives, olive oils \\
\hline Sugars, sweets and desserts & $\begin{array}{l}\text { Sugars, candies, Iranian confectioneries (gaz, sohan, noghl), jam, jelly, honey, chocolates, cookies, cakes, } \\
\text { confections, caramels }\end{array}$ \\
\hline Tea and coffee & Tea, coffee \\
\hline
\end{tabular}

\section{Red meats}

Organ meats

mish and other seafood

Eggs

Carbonated drinks 
the two methods, we can use the two (DR/FFQ) interchangeably. The limits of agreement were given as 1.96 times the standard deviation of the difference. Bland and Altman stated: 'The limits of agreement are only estimates of the values which apply to the whole population. We might sometimes wish to use standard errors and CI to see how precise our estimates are, provided the differences follow a distribution which is approximately Normal' ${ }^{,(27)}$. Finally, in order to evaluate the long-term stability, factor scores were split into quintiles. Then, the agreement of subjects' quintiles classifications by factor scores between baseline and after 8 years of follow-up was calculated and the proportions of subjects at the same quintile, adjacent quintile and opposite quintile were presented. The weighted $k$ statistics was calculated. $P$ values $<0.05$ were considered significant.

\section{Results}

The mean age of the participants was $35 \cdot 6$ (SD 16.8) years, $39 \cdot 8$ (SD 18.8) years for men and 33.4 (SD 15.4) years for women; mean BMI was 25.5 (SD 5.2$) \mathrm{kg} / \mathrm{m}^{2}, 24.7$ (SD 3.8 ) $\mathrm{kg} / \mathrm{m}^{2}$ for men and $26.0(\mathrm{SD} 5.8) \mathrm{kg} / \mathrm{m}^{2}$ for women. Characteristics of the study participants and the TLGS population have been reported in a previous study ${ }^{(12)}$. Median daily energy-adjusted consumption of the nineteen food groups assessed with FFQ and $\mathrm{mDR}$ are presented in Table 2. Some food groups estimated in FFQ1 were higher than their estimates in FFQ2; these included fast foods, eggs, carbonated drinks, refined grains, vegetable oil, animal fat and fast foods. In contrast, estimated animal fats and olives were lower by the FFQ1 in comparison with FFQ2. On the other hand, eleven food groups estimated in FFQ2 were higher, and legumes and nuts, carbonated drinks, red meats and potatoes in FFQ2 were lower than in the $\mathrm{mDR}$. The median estimates of organ meats, poultry, carbonated drinks and dairy products according to FFQ3 were higher than those based on FFQ2. However, median estimates of red meat, eggs, potatoes, refined grains, salty snacks, vegetable oil and sugars were lower than those based on FFQ2. Spearman correlations of daily intakes of the food groups extracted from the FFQ2, FFQ3 and mDR are listed in Table 3. Spearman correlation coefficients ranged from -0.27 for legumes to 0.70 for tea and coffee (mean 0.40) for the comparison between FFQ2 and the mDR. The highest correlation between FFQ2 and FFQ3 was found for animal fats (0.39) and the lowest correlation for poultry $(0 \cdot 10)$, with the mean of $0 \cdot 28$.

Using factor analysis, two major dietary patterns were extracted for FFQ1, FFQ2 and mDR, and three major dietary patterns were extracted for FFQ3. The factor loadings for each pattern derived from FFQ and $\mathrm{mDR}$ are shown in Table 4. Overall, the total percentage of variance explained by the major dietary patterns derived from FFQ1, FFQ2, FFQ3 and the mDR, was $27 \cdot 4,31 \cdot 6,39 \cdot 0$ and $32 \cdot 0 \%$, respectively. Thus, two relatively similar patterns, 'Iranian Traditional' and 'Western', were identified from all four sources and a 'Combined' pattern was also identified for FFQ3. The 'Iranian Traditional' was highly loaded for vegetables, fruits, potatoes, dairy products, legumes and nuts, whole grains, tea and coffee, olive, eggs, red meat and organ meat, the 'Western' was highly loaded for carbonated drinks, salty snacks and salty vegetables, sugars, sweets, desserts, vegetable oil, animal fat, fast foods, poultry, fish and other seafood and

Table 2. Daily energy-adjusted intakes of nineteen food groups estimated with the mean of twelve 24-h dietary recalls (mDR) and three FFQ, during 8 years of follow-up: Tehran Lipid and Glucose Study

(Median and interquartile ranges)

\begin{tabular}{|c|c|c|c|c|c|c|c|c|}
\hline \multirow[b]{2}{*}{ Food groups (g/d) } & \multicolumn{2}{|c|}{ mDR† (n 132) } & \multicolumn{2}{|c|}{ FFQ1† (n 132) } & \multicolumn{2}{|c|}{ FFQ2† (n 132) } & \multicolumn{2}{|c|}{ FFQ3† ( $n$ 89) } \\
\hline & Median & $\begin{array}{l}\text { Interquartile } \\
\text { range }\end{array}$ & Median & $\begin{array}{l}\text { Interquartile } \\
\text { range }\end{array}$ & Median & $\begin{array}{l}\text { Interquartile } \\
\text { range }\end{array}$ & Median & $\begin{array}{l}\text { Interquartile } \\
\text { range }\end{array}$ \\
\hline Red meats & $30 \cdot 9^{\star \star}$ & $18 \cdot 1$ & $25 \cdot 9$ & $20 \cdot 4$ & $25 \cdot 4$ & $19 \cdot 5$ & $17 \cdot 0^{\star \star}$ & $14 \cdot 8$ \\
\hline Organ meats & $0.05^{\star \star}$ & 0.2 & $1 \cdot 0$ & 1.4 & $1 \cdot 3$ & 1.5 & $1 \cdot 5^{\star}$ & 1.9 \\
\hline Poultry, fish and other seafood & $25 \cdot 3$ & $21 \cdot 8$ & $19 \cdot 7$ & $21 \cdot 6$ & $22 \cdot 8$ & $20 \cdot 3$ & $32 \cdot 2^{*}$ & $20 \cdot 7$ \\
\hline Fast foods & $4 \cdot 0^{\star *}$ & $7 \cdot 6$ & $17 \cdot 9^{*}$ & $20 \cdot 5$ & $16 \cdot 1$ & $17 \cdot 0$ & $13 \cdot 2$ & $14 \cdot 7$ \\
\hline Eggs & $15 \cdot 8$ & $11 \cdot 6$ & $17 \cdot 1^{*}$ & $15 \cdot 1$ & $15 \cdot 2$ & $12 \cdot 0$ & $9 \cdot 3^{\star *}$ & $11 \cdot 8$ \\
\hline Carbonated drinks & $63 \cdot 0^{\star \star}$ & $65 \cdot 1$ & $34 \cdot 9^{\star *}$ & $54 \cdot 1$ & $23 \cdot 3$ & $46 \cdot 2$ & $32 \cdot 6^{*}$ & $45 \cdot 2$ \\
\hline Dairy products & $202 \cdot 5^{\star \star}$ & $124 \cdot 7$ & $315 \cdot 9$ & $174 \cdot 3$ & $302 \cdot 8$ & $166 \cdot 9$ & $416 \cdot 6^{\star \star}$ & $277 \cdot 8$ \\
\hline Fruits and dried fruits & $217 \cdot 3^{\star \star}$ & $138 \cdot 6$ & $413 \cdot 7$ & 237.6 & $388 \cdot 7$ & $296 \cdot 1$ & $321 \cdot 0$ & 257.0 \\
\hline Vegetables & $203 \cdot 7$ & $98 \cdot 1$ & $219 \cdot 8$ & 143.9 & $215 \cdot 1$ & $126 \cdot 5$ & $265 \cdot 1$ & $221 \cdot 8$ \\
\hline Legumes and nuts & $34 \cdot 0^{* *}$ & $22 \cdot 2$ & $33 \cdot 8$ & 35.7 & $29 \cdot 2$ & $24 \cdot 2$ & $30 \cdot 6$ & 33.5 \\
\hline Potatoes & $24 \cdot 0^{\star *}$ & $17 \cdot 9$ & $12 \cdot 8$ & $17 \cdot 8$ & $17 \cdot 2$ & $17 \cdot 7$ & $10 \cdot 0^{* *}$ & $12 \cdot 3$ \\
\hline Whole grains & $63 \cdot 9^{\star \star}$ & $43 \cdot 1$ & $67 \cdot 6$ & $71 \cdot 3$ & $76 \cdot 6$ & 74.9 & $67 \cdot 8$ & $81 \cdot 32$ \\
\hline Refined grains & $328 \cdot 1^{\star *}$ & $77 \cdot 4$ & $458 \cdot 0^{\star \star}$ & $164 \cdot 3$ & 360.9 & $141 \cdot 0$ & $280 \cdot 1^{\star \star}$ & $180 \cdot 2$ \\
\hline Salty snacks and vegetables & $16 \cdot 5^{\star \star}$ & $15 \cdot 3$ & $26 \cdot 2$ & $25 \cdot 5$ & $28 \cdot 9$ & $24 \cdot 7$ & $16 \cdot 8^{\star \star}$ & $20 \cdot 5$ \\
\hline Animal fats & $2 \cdot 8^{\star}$ & $4 \cdot 8$ & $0.22^{\star \star}$ & $7 \cdot 2$ & $3 \cdot 8$ & $6 \cdot 7$ & 4.5 & $6 \cdot 1$ \\
\hline Vegetable oils & $28 \cdot 8$ & $11 \cdot 4$ & $64 \cdot 4^{\star \star}$ & $50 \cdot 4$ & $27 \cdot 8$ & $17 \cdot 3$ & $18 \cdot 1^{\star \star}$ & $18 \cdot 3$ \\
\hline Olives & $0.07^{\star *}$ & $0 \cdot 2$ & $0.5^{\star \star}$ & 1.5 & 0.8 & 1.8 & 0.7 & $2 \cdot 7$ \\
\hline Sugars, sweets and desserts & $47 \cdot 1^{\star \star}$ & $23 \cdot 6$ & 51.9 & $29 \cdot 4$ & $53 \cdot 4$ & $36 \cdot 7$ & $29 \cdot 1^{\star \star}$ & $23 \cdot 8$ \\
\hline Tea and coffee & $546 \cdot 2^{\star *}$ & $451 \cdot 4$ & $613 \cdot 3$ & $581 \cdot 6$ & $578 \cdot 6$ & $503 \cdot 8$ & 546.5 & $558 \cdot 9$ \\
\hline
\end{tabular}

Medians of energy-adjusted food group intakes were significantly different from FFQ2: ${ }^{*} P<0.05,{ }^{\star \star} P<0.01$ (Wilcoxon test).

$\dagger$ Food intakes were energy-adjusted by the residual method. A total of twelve $24 \mathrm{~h}$ dietary recalls (DR) were collected from each subject on a consecutive monthly basis. The first FFQ was completed 1 month before collection of the first $24 \mathrm{~h}$ DR (FFQ1), the second, 1 month after the last $24 \mathrm{~h}$ DR (FFQ2) and the third one at the end of 8 years of follow-up (FFQ3). 
Table 3. Spearman correlation coefficients of nineteen food groups estimated with the mean of twelve $24 \mathrm{~h}$ dietary recalls (mDR) and two FFQ, during 8 years of follow-up: Tehran Lipid and Glucose Study*

\begin{tabular}{lccccc}
\hline & \multicolumn{2}{c}{ FFQ2 $v$. mDR $(n$ 132) } & & \multicolumn{2}{c}{ FFQ2 $v$. FFQ3 $(n$ 89) } \\
\cline { 2 - 3 } \cline { 5 - 6 } Food groups $(\mathrm{g} / \mathrm{d})$ & Crude & Energy adjusted & & Crude & Energy adjusted \\
\hline Red meats & 0.46 & 0.30 & & 0.24 & 0.21 \\
Organ meats & 0.37 & 0.33 & & 0.67 & 0.50 \\
Poultry, fish and other seafood & 0.44 & 0.35 & & 0.27 & 0.10 \\
Fast foods & 0.35 & 0.35 & & 0.53 & 0.34 \\
Eggs & 0.40 & 0.29 & & 0.40 & 0.32 \\
Carbonated drinks & 0.43 & 0.40 & & 0.50 & 0.23 \\
Dairy products & 0.53 & 0.50 & & 0.29 & 0.18 \\
Fruits and dried fruits & 0.41 & 0.30 & & 0.35 & 0.26 \\
Vegetables & 0.54 & 0.51 & & 0.25 & 0.27 \\
Legumes and nuts & 0.33 & 0.27 & & 0.29 & 0.20 \\
Potatoes & 0.30 & 0.29 & & 0.32 & 0.25 \\
Whole grains & 0.52 & 0.50 & & 0.19 & 0.25 \\
Refined grains & 0.59 & 0.52 & & 0.41 & 0.38 \\
Salty snacks and vegetables & 0.46 & 0.46 & & 0.32 & 0.25 \\
Animal fats & 0.43 & 0.38 & & 0.52 & 0.39 \\
Vegetable oils & 0.41 & 0.38 & & 0.29 & 0.18 \\
Olives & 0.50 & 0.48 & & 0.44 & 0.23 \\
Sugars, sweets and desserts & 0.52 & 0.37 & & 0.40 & 0.34 \\
Tea and coffee & 0.79 & 0.70 & & 0.43 & 0.42 \\
\hline
\end{tabular}

${ }^{*}$ Food intakes were energy-adjusted by the residual method; all $P<0.0001$. A total of twelve $24 \mathrm{~h}$ dietary recalls (DR) were collected on a consecutive monthly basis. The second FFQ was completed 1 month after collection of the last $24 \mathrm{~h}$ DR (FFQ2) and the third one at the end of 8 years of follow-up (FFQ3).

refined grains, and the 'Combined' factor was highly loaded for potatoes, tea and coffee, vegetable oils, eggs, legumes and nuts, sugars, whole grains and salty snacks.

Intra-class correlation coefficients between FFQ1 and FFQ2 were $0.72(P<0.001)$ for the Iranian Traditional pattern and $0.80(P<0.001)$ for the Western pattern; coefficients between FFQ2 and FFQ3 were $-0.09(P=0.653)$ for the Iranian Traditional and $0.49(P<0 \cdot 001)$ for the Western pattern
(Table 5). Correlation coefficients corrected for monthto-month variation in the DR were 0.48 for the Iranian Traditional pattern and 0.75 for the Western pattern. Table 6 shows the correlation between the factor scores of two major dietary patterns and mean nutrient intakes derived from the DR. The Iranian Traditional pattern was positively and the Western pattern was negatively correlated with intakes of all nutrients shown in the table, except for total fat and

Table 4. Factor-loading matrix for the major dietary patterns identified from means of twelve 24-h dietary recalls (mDR) and three FFQ developed for the Tehran Lipid and Glucose Study* $\dagger$

\begin{tabular}{|c|c|c|c|c|c|c|c|c|c|}
\hline \multirow[b]{2}{*}{ Food groups } & \multicolumn{2}{|c|}{ FFQ1 ( $n$ 132) } & \multicolumn{2}{|c|}{ FFQ2 ( $n$ 132) } & \multicolumn{2}{|c|}{$\operatorname{mDR}(n 132)$} & \multicolumn{3}{|c|}{ FFQ3 $(n 89)$} \\
\hline & $\begin{array}{c}\text { Iranian } \\
\text { Traditional }\end{array}$ & Western & $\begin{array}{l}\text { Iranian } \\
\text { Traditional }\end{array}$ & Western & $\begin{array}{l}\text { Iranian } \\
\text { Traditional }\end{array}$ & Western & $\begin{array}{l}\text { Iranian } \\
\text { Traditional }\end{array}$ & Western & Combined \\
\hline Vegetables & 0.73 & - & 0.71 & - & 0.54 & - & - & -0.41 & - \\
\hline Potatoes & 0.65 & - & 0.39 & - & 0.63 & 0.24 & - & - & 0.69 \\
\hline Eggs & 0.59 & - & 0.74 & 0.23 & 0.69 & - & - & - & 0.54 \\
\hline Red meat & 0.59 & 0.22 & 0.56 & 0.35 & 0.42 & 0.39 & 0.55 & - & - \\
\hline Fruit & 0.58 & 0.20 & 0.21 & - & 0.36 & - & 0.68 & - & - \\
\hline Dairy products & 0.48 & 0.25 & 0.22 & - & 0.33 & 0.30 & 0.74 & - & - \\
\hline Whole grains & 0.36 & - & 0.33 & - & 0.38 & - & - & - & 0.42 \\
\hline Tea and coffee & 0.29 & - & 0.42 & 0.39 & 0.35 & 0.29 & - & - & 0.67 \\
\hline Organ meats & 0.26 & - & 0.49 & - & 0.35 & - & 0.44 & 0.27 & - \\
\hline Legumes and nuts & 0.20 & - & 0.61 & - & 0.54 & - & 0.43 & -0.20 & 0.43 \\
\hline Carbonated drinks & - & 0.64 & - & 0.71 & - & 0.76 & 0.54 & 0.33 & - \\
\hline Salty snacks and vegetables & 0.20 & 0.60 & 0.54 & 0.32 & 0.30 & 0.33 & 0.40 & - & 0.32 \\
\hline Sugars, sweets and desserts & - & 0.54 & - & 0.62 & - & 0.63 & 0.36 & - & 0.44 \\
\hline Vegetable oils & - & 0.52 & 0.37 & 0.45 & 0.50 & 0.59 & - & 0.47 & 0.58 \\
\hline Animal fats & - & 0.51 & - & 0.55 & - & 0.35 & 0.30 & 0.49 & - \\
\hline Fast foods & - & 0.48 & - & 0.52 & - & 0.45 & 0.66 & - & - \\
\hline Poultry, fish and other seafood & - & 0.44 & - & 0.52 & - & 0.54 & 0.70 & - & - \\
\hline Olives & 0.33 & - & 0.20 & - & 0.45 & - & - & -0.42 & - \\
\hline Refined grains & - & 0.20 & - & 0.56 & 0.36 & 0.67 & - & 0.72 & - \\
\hline
\end{tabular}

* A total of twelve 24-h dietary recalls (DR) were collected on a consecutive monthly basis. The first FFQ was completed 1 month before collection of the first 24-h DR (FFQ1), the second, 1 month after the last $24 \mathrm{~h}$ DR (FFQ2) and the third one at the end of 8 years of follow-up (FFQ3).

$\dagger$ Absolute values $<0.2$ were excluded from the table for simplicity. 
Table 5. Correlation coefficients for the pattern scores derived from the mean of twelve $24 \mathrm{~h}$ dietary recalls (mDR) and the three FFQ developed for the Tehran Lipid and Glucose Study†

\begin{tabular}{|c|c|c|c|c|}
\hline & \multicolumn{2}{|c|}{ Iranian Traditional pattern } & \multicolumn{2}{|c|}{ Western pattern } \\
\hline & Crude & Corrected $\ddagger$ & Crude & Corrected \\
\hline FFQ1 v. FFQ2§ & $0.72^{\star *}$ & - & $0.80^{* *}$ & - \\
\hline FFQ2 v. FFQ3§ & -0.09 & - & $0.49^{\star \star}$ & - \\
\hline FFQ2 v. mDR $\|$ & $0.48^{\star \star}$ & 0.48 & $0.74^{\star \star}$ & 0.75 \\
\hline
\end{tabular}

** $P<0.001$

† A total of twelve $24 \mathrm{~h}$ dietary recalls (DR) were collected on a consecutive monthly basis ( $n$ 132). The first FFQ was completed 1 month before collection of the first $24 \mathrm{~h}$ DR (FFQ1, $n$ 132), the second, 1 month after the last $24 \mathrm{~h}$ DR (FFQ2, $n 132$ ) and the third one at the end of 8 years of follow-up (FFQ3, $n$ 89).

$\ddagger$ Corrected for month-to-month variation in DR.

$\S$ Intra-class correlation.

॥V Values are Spearman correlation coefficients.

saturated fat that were negatively correlated with the Iranian pattern but positively correlated with the Western pattern.

The stability for the scores of two factors (Iranian Traditional and Western) was assessed over 8 years (Table 7). The percentage of subjects at the same quintile was higher for the Western dietary pattern in comparison to the Iranian Traditional pattern $(27 \cdot 1 \% v \cdot 20 \cdot 2 \%)$; however, the proportion of individuals at the opposite quintile was reversed $(35.8 \% \mathrm{v}$. $41.5 \%)$. The weighted $k$ statistics for the Iranian Traditional and the Western dietary patterns were 0.09 (95\% CI - 0.05, $0 \cdot 23)$ and $0 \cdot 20(95 \%$ CI 0.05, 0.34), respectively.

The agreement (intra-class correlation) between the factor scores of dietary patterns derived from FFQ2 and mDR was 0.79 for the Iranian Traditional dietary pattern and 0.86 for the Western dietary pattern. In Fig. 1, the Bland-Altman plots of the Iranian Traditional dietary pattern (a) and the Western dietary pattern (b) are presented. The 95\% limits of agreement for the difference between the factor scores obtained from $\mathrm{FFQ} 2$ and $\mathrm{mDR}$ lay between -1.58 and
+1.58 for the Iranian Traditional dietary pattern; and between -1.33 and +1.33 for the Western dietary pattern.

\section{Discussion}

In the present study, using factor analysis, two major dietary patterns were extracted, i.e. the 'Iranian Traditional' and 'Western' dietary pattern at baseline. These two derived patterns were relatively similar, both in dietary data obtained from the FFQ and in the means of twelve DR. The strong correlations among FFQ1 and FFQ2 suggested a high reliability; and the correlation coefficient between FFQ2 and the mDR as a reference method, ranged from 0.48 to 0.75 for those patterns, indicated a reasonable validity of FFQ2 against the $\mathrm{mDR}$ in defining major dietary patterns. Overall, three major dietary patterns were obtained after long-term follow-up, including 'Iranian Traditional', 'Western', which had relatively similar food groups to the baseline extracted patterns, and a new pattern or the 'Combined' pattern. The Western dietary pattern showed good long-term stability; the non-significant correlation of the Iranian Traditional pattern, however, suggests instability of the mentioned pattern over time.

Few studies have assessed the reliability and validity of dietary patterns, especially in Asian countries; however, because of socio-economic, cultural and ethnic differences and diversity in food preferences and access to various foods, such investigations seem necessary in other Middle Eastern populations. The first derived pattern, or the 'Iranian Traditional' one, reflected the common eating habits of Iranian people, high in vegetables, fruits, potatoes, dairy products, legumes and nuts, whole grains, tea and coffee, olive, eggs, red and organ meats; this pattern contained almost all healthy food items except red and organ meat; this is mainly because in Iran, more traditional foods are prepared with red meat and eggs along with vegetables and legumes like stews 'Khoresh'

Table 6. Spearman correlation coefficients between the Iranian Traditional and Western dietary pattern scores derived from means of twelve $24 \mathrm{~h}$ dietary recalls (mDR) and FFQ and mean nutrients intake obtained from dietary recalls (DR) $(n 132)$

\begin{tabular}{|c|c|c|c|c|c|c|}
\hline \multirow{2}{*}{$\begin{array}{l}\text { Energy-adjusted } \\
\text { nutrients from } \mathrm{DR}^{\star}\end{array}$} & \multicolumn{3}{|c|}{ Iranian Traditional dietary pattern } & \multicolumn{3}{|c|}{ Western dietary pattern } \\
\hline & FFQ1 & FFQ2 & $\mathrm{mDR}$ & FFQ1 & FFQ2 & $\mathrm{mDR}$ \\
\hline Carbohydrate & 0.13 & 0.10 & 0.04 & -0.21 & -0.08 & -0.10 \\
\hline Protein & 0.05 & 0.10 & 0.14 & -0.08 & -0.05 & -0.07 \\
\hline Total fat & -0.13 & -0.11 & -0.07 & 0.20 & 0.06 & 0.09 \\
\hline Saturated fat & -0.08 & -0.16 & -0.24 & $0 \cdot 16$ & 0.03 & 0.04 \\
\hline Fibre & 0.04 & 0.13 & 0.17 & -0.31 & -0.23 & -0.33 \\
\hline $\mathrm{Ca}$ & 0.06 & 0.19 & 0.15 & -0.27 & -0.34 & -0.40 \\
\hline $\mathrm{Mg}$ & 0.34 & 0.39 & 0.41 & -0.41 & -0.33 & -0.39 \\
\hline $\mathrm{K}$ & 0.24 & 0.33 & 0.34 & -0.33 & -0.29 & -0.37 \\
\hline $\mathrm{P}$ & 0.16 & 0.21 & 0.20 & -0.25 & -0.29 & -0.31 \\
\hline $\mathrm{Zn}$ & 0.22 & 0.29 & 0.40 & $-0 \cdot 19$ & -0.11 & -0.21 \\
\hline Retinol & $0 \cdot 10$ & 0.15 & 0.29 & -0.15 & -0.16 & -0.22 \\
\hline$\beta$-Carotene & 0.19 & 0.25 & 0.25 & -0.24 & -0.15 & -0.22 \\
\hline$\alpha$-Tocopherol & 0.10 & 0.14 & 0.20 & -0.08 & -0.13 & -0.07 \\
\hline Vitamin E & 0.13 & 0.12 & 0.16 & -0.01 & -0.11 & -0.05 \\
\hline Vitamin C & 0.27 & 0.26 & 0.21 & -0.22 & -0.21 & -0.19 \\
\hline Thiamine & 0.10 & 0.17 & 0.20 & -0.34 & -0.22 & -0.25 \\
\hline Riboflavin & 0.13 & 0.23 & 0.28 & -0.18 & -0.26 & -0.35 \\
\hline
\end{tabular}

* A total of twelve $24 \mathrm{~h}$ DR were collected on a consecutive monthly basis. The first FFQ was completed 1 month before collection of the first $24 \mathrm{~h}$ DR (FFQ1) and the second, 1 month after the last $24 \mathrm{~h}$ DR (FFQ2). 
Table 7. Agreement of the classification to the same, adjacent or opposite quintiles for the Iranian Traditional and Western dietary pattern scores derived from the second FFQ (FFQ2) and third FFQ (FFQ3) $(n 89)^{*}$

\begin{tabular}{lccccr}
\hline & Same quintile (\%) & Adjacent quintile (\%) & Opposite quintile (\%) & Weighted $k$ & $95 \% \mathrm{Cl}$ \\
\hline Iranian Traditional & 20.2 & 38.3 & 41.5 & 0.09 & $-0.05,0.23$ \\
Western & 27.1 & 37.1 & 35.8 & 0.20 & $0.05,0.34$ \\
\hline
\end{tabular}

*The FFQ2 was completed 1 month after collection of the last $24 \mathrm{~h}$ dietary recalls at baseline, and the FFQ3 at the end of 8 years of follow-up.

(dish of red meat and vegetables served with rice) and different kinds of 'omelettes' (a dish made of eggs with onion, potato, tomato and pepper). The second factor, loaded mainly for the Western food groups, was the 'Western pattern' (high in carbonated drinks, salty snacks and salty vegetables, sugars, sweets, desserts, vegetable oil, animal fat, fast foods, poultry, fish and other seafood and refined grains). Similar to the Western dietary pattern derived from the Australian adolescents cohort ${ }^{(28)}$, poultry and fish were highly loaded for the Western pattern because in Iranian recipes, they are most commonly prepared by frying and are usually consumed with the skin. Furthermore, total fat and saturated fat intake, the impairing effects of which on human health have been described elsewhere ${ }^{(29,30)}$, were positively correlated with the Western pattern. For both dietary patterns, factor loadings of the FFQ and mDR food items were partly different; as $\mathrm{Hu}$ et $a l^{(1)}$ elaborated on it, this was a result of methodological differences between the dietary assessment methods, random statistical variations and different assessment periods ${ }^{(1)}$; nevertheless, the major patterns extracted from the FFQ and DR were comparable.

The correlations of the dietary patterns between FFQ1 and FFQ2 for the Iranian Traditional and the Western pattern revealed a good reliability; and the correlations of the dietary patterns between FFQ2 and the $\mathrm{mDR}$ for the Iranian Traditional and the Western pattern represented a reasonable comparative validity of dietary patterns derived by factor analysis using the data of FFQ in an Iranian population. For reducing the effect of difference in seasonal food availability, twelve DR (one for each month) were collected, which cover variability in food consumption during different seasons. Also, the deattenuated validity correlations were calculated to correct month-to-month within-person variations. Although the methods of comparative validity and reliability of dietary patterns were different, the obtained correlations of the present study were similar to those documented by other studies, and ranged between 0.45 and 0.74 in American men ${ }^{(1)}$ for the prudent and Western dietary patterns; 0.41 and 0.73 for healthy, Western and drinker patterns in Swedish women ${ }^{(9)}$; 0.34 and 0.61 for green, sweet and traditional patterns for Danish men and women ${ }^{(4)} ; 0.35$ and 0.67 for prudent and Western patterns for pregnant British women ${ }^{(10)}$ and 0.36 and 0.62 for healthy, Western and traditional Japanese patterns in Japanese men and women ${ }^{(7)}, 0.45$ and 0.36 for healthy and Western pattern in Australian adolescents ${ }^{(28)}$.

The complex nature of the Iranian Traditional dietary pattern makes it very difficult to interpret. Because of worldwide differences in geographical, cultural and methodological variations (sampling, food grouping, number of variables used in factor analysis), interpretations of dietary pattern analyses should be made with caution ${ }^{(9)}$. Overall, the Western pattern of the present study is similar to 'Western' dietary patterns in the Health Professionals' Follow-up Study ${ }^{(1)}$, the Swedish ${ }^{(9)}$, the American women ${ }^{(31)}$, Spanish men and women participating in the Spanish SUN project ${ }^{(32)}$ and Australian adolescents ${ }^{(28)}$ Besides, a rational agreement was observed in our study between the FFQ2 and $\mathrm{mDR}$ scores, which was in accordance with the results of previous studies ${ }^{(1,7,9,10)}$.
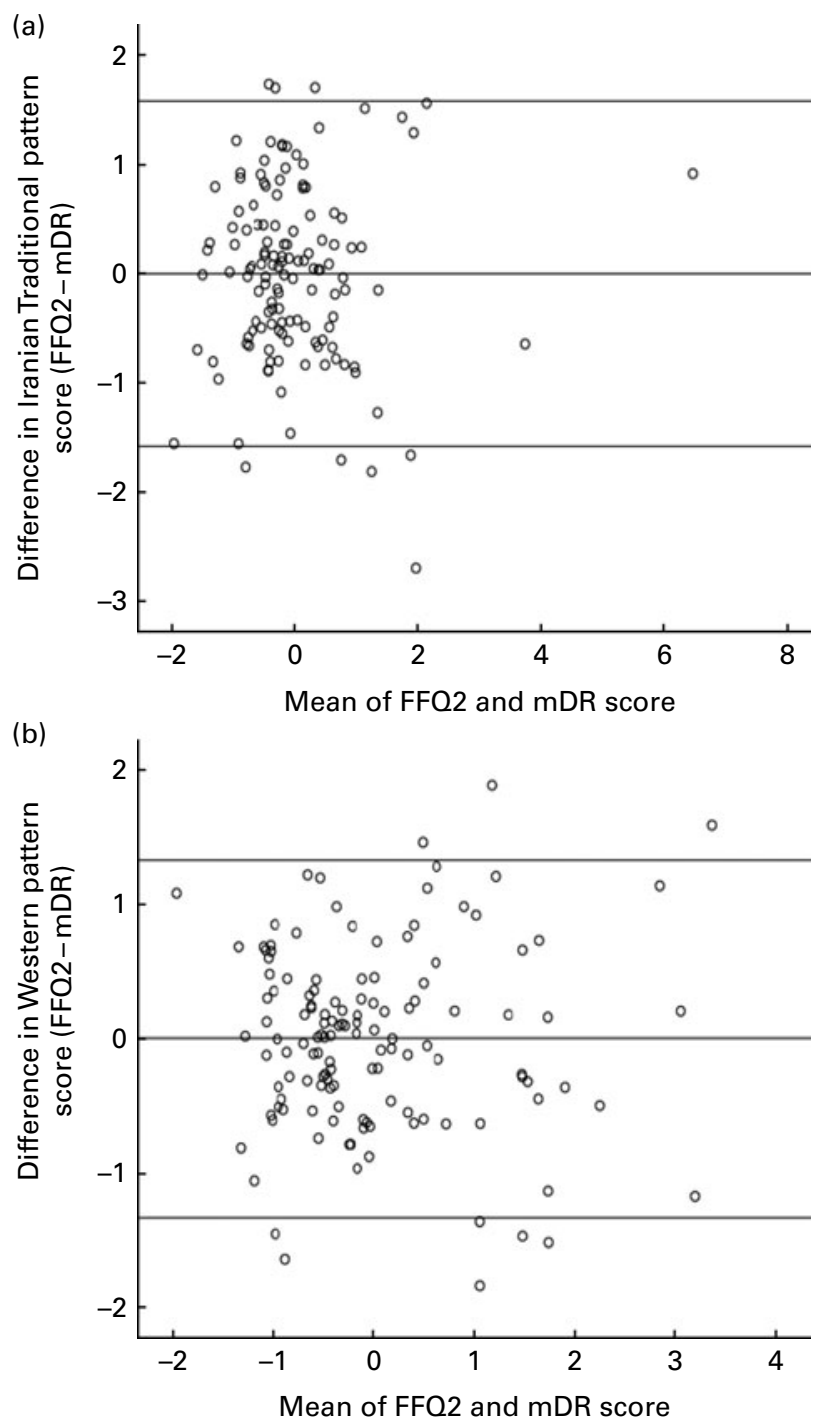

Fig. 1. Bland-Altman plots for agreement between (a) Iranian Traditional and (b) Western pattern scores derived from the second FFQ (FFQ2) and mean of twelve dietary records (mDR) among 132 participants of the Tehran Lipid and Glucose Study. 
The correlation between dietary pattern scores obtained from $\mathrm{mDR}$ and FFQ with specific mean nutrient intakes, obtained from twelve $24 \mathrm{~h} \mathrm{DR}$, indicated the face validity of principal component analysis in our population, an association which was expected. Also, the associations of dietary patterns derived from mDR used as a 'gold standard' and nutrient intakes were higher than those based on the use of FFQ, a finding in agreement with that of the Hu et al. study ${ }^{(1)}$.

Relatively limited numbers of studies have evaluated the long-term stability of dietary patterns ${ }^{(3,8,34)}$. Similarly, Northstone et $a l .{ }^{(3)}$ found that four dietary patterns over a 4 -year post-partum follow-up in women were mostly consistent, with the exception of loss of the 'Traditional' one, which indicates that the exact comparison could not be made ${ }^{(3)}$. In another study, three patterns were consistently extracted at four time points; however, an extra 'snack' pattern was derived at the baseline and 'health conscious' pattern was modified at the end of follow-up ${ }^{(33)}$. In contrast, in a study of middle-aged Swedish women, there was no evidence for the stability of dietary patterns after 7 years, indicating that dietary intakes should be updated at least every 7 years ${ }^{(8)}$ Our findings suggest that over an 8-year follow-up in an Iranian population, dietary patterns were relatively unstable, with the exception of the Western pattern (intra-class correlation $=0.49 ; P<0.001)$. The variations in the stability of dietary patterns can be explained by differences in follow-up duration, age of participants (childhood, adulthood) and physiological status (growth, pregnancy, etc). Also, nutrition transition is an important issue in losing and introducing patterns over time, in developing countries like Iran.

Regarding the study limitations, first, we considered DR as a 'gold standard' for assessing the comparative validity of the FFQ in its description of dietary patterns. The measurement errors in DR and potential changes in eating behaviours are inevitable ${ }^{(22)}$. Second, limitations of the FFQ (such as measurement errors including selective under- or overreporting of intakes of certain foods, relying on memory, requiring skilled professionals for the interview) ${ }^{(33)}$ for assessing dietary intakes should be taken into account. Third, there are some limitations in the factor analysis method, namely several subjective or arbitrary decisions in the use of factor analysis including the food grouping, number of factors, rotation method and interpretability of factors ${ }^{(34)}$. Fourth, due to the small sample size ( $n$ 132), we could not separate men and women to define the major dietary pattern of each sex. Fifth, the total variance explained by two dietary patterns derived from FFQ1, FFQ2 and mDR was 27.41, 31.65 and $32.04 \%$, respectively; hence, these two patterns are not representative of all of our patterns available; this despite the fact that other minor variables were less interpretable. Finally, using the FCT is another limitation of our study. Not having a comprehensive Iranian FCT with which to compare intakes, we do not know how this affects our results concerning the correlation of dietary pattern scores and nutrients. The strength of this study was the fact that since there were no differences between the characteristics of those subjects who participated in this study and the participants of the TLGS, the results can be generalised to the cohort population.

\section{Conclusion}

Our data indicate reasonable reliability and validity of the derived dietary patterns defined by factor analysis using data from the FFQ, which is a suitable approach for determining the dietary patterns of Iranians and for studying the relationship between dietary patterns and health outcomes in epidemiological studies. Although the Western pattern was found to be fairly stable, the Iranian Traditional pattern was mostly unstable over the 8 years of the study period.

\section{Acknowledgements}

The authors would like to thank the subjects who participated in the present study. We are grateful to the staff of the nutrition department. We would like to thank Ms N. Shiva for the language editing of the manuscript. This study was part of the TLGS and was supported by the Research Institute of Endocrine Sciences, Shahid Beheshti University of Medical Sciences, Islamic Republic of Iran (grant no. 089). G. A., A. R., F. H.-E. and P. M. designed the study, collected and analysed the data, and wrote the manuscript. Y. M. supervised the final edition of the statistical analysis. F.A. supervised the study. None of the authors had any personal or financial conflicts of interest.

\section{References}

1. Hu FB, Rimm E, Smith-Warner SA, et al. (1999) Reproducibility and validity of dietary patterns assessed with a food-frequency questionnaire. Am J Clin Nutr 69, 243-249.

2. Hu FB (2002) Dietary pattern analysis: a new direction in nutritional epidemiology. Curr Opin Lipidol 13, 3-9.

3. Northstone K \& Emmett PM (2008) A comparison of methods to assess changes in dietary patterns from pregnancy to 4 years post-partum obtained using principal components analysis. Br J Nutr 99, 1099-1106.

4. Togo P, Heitmann BL, Sorensen TI, et al. (2003) Consistency of food intake factors by different dietary assessment methods and population groups. Br J Nutr 90, 667-678.

5. Newby PK, Muller D, Hallfrisch J, et al. (2004) Food patterns measured by factor analysis and anthropometric changes in adults. Am J Clin Nutr 80, 504-513.

6. Schatzkin A, Kipnis V, Carroll R, et al. (2003) A comparison of a food frequency questionnaire with a 24-hour recall for use in an epidemiological cohort study: results from the biomarker-based Observing Protein and Energy Nutrition (OPEN) study. Int J Epidemiol 32, 1054-1062.

7. Okubo H, Murakami K, Sasaki S, et al. (2010) Relative validity of dietary patterns derived from a self-administered diet history questionnaire using factor analysis among Japanese adults. Public Health Nutr 13, 1080-1089.

8. Weismayer C, Anderson JG \& Wolk A (2006) Changes in the stability of dietary patterns in a study of middle-aged Swedish women. J Nutr 136, 1582-1587.

9. Rashid Khani B, Ye W, Terry P, et al. (2004) Reproducibility and validity of major dietary patterns among Swedish women assessed with a food-frequency questionnaire. J Nutr 134, 1541-1545.

10. Crozier SR, Inskip HM, Godfrey KM, et al. (2008) Dietary patterns in pregnant women: a comparison of food-frequency questionnaires and $4 \mathrm{~d}$ prospective diaries. Br J Nutr 99, 869-875. 
11. Mirmiran P, Esfahani FH, Mehrabi Y, et al. (2010) Reliability and relative validity of an FFQ for nutrients in the Tehran lipid and glucose study. Public Health Nutr 13, 654-662.

12. Esfahani FH, Asghari G, Mirmiran P, et al. (2010) Reproducibility and relative validity of food group intake in a food frequency questionnaire developed for the Tehran Lipid and Glucose Study. I Epidemiol 20, 150-158.

13. Azizi F, Rahmani M, Emami H, et al. (2002) Cardiovascular risk factors in an Iranian urban population: Tehran Lipid and Glucose Study (phase 1). Soz Praventivmed 47, 408-426.

14. Kimiagar M (1995) National Food Consumption Survey. Teharan: National Nutrition and Food Technology Research Institute.

15. Mirmiran P, Azadbakht L \& Azizi F (2006) Dietary diversity within food groups: an indicator of specific nutrient adequacy in Tehranian women. J Am Coll Nutr 25, 354-361.

16. Willett W \& Lenart E (1998) Reproducibility and validity of food frequency questionnaires. In Nutritional Epidemiology, 2nd ed. [W Willett, editor]. New York: Oxford University Press.

17. Ghafarpour M, Houshiar-Rad A \& Kianfar H (1999) The Manual for Household Measures, Cooking Yields Factors and Edible Portion of Food. Tehran: Keshavarzi Press.

18. Azar M \& Sarkisian E (1980) Food Composition Table of Iran. Tehran: National Nutrition and Food Research Institute of Shaheed Beheshti University.

19. US Department of Agriculture Agricultural Research Service (2011) Nutrient Data. http://www.ars.usda.gov/main/ site main.htm? modecode $=12-35-45-00$

20. Esmaillzadeh A \& Azadbakht L (2008) Major dietary patterns in relation to general obesity and central adiposity among Iranian women. J Nutr 138, 358-363.

21. Rezazadeh A, Rashidkhani B \& Omidvar N (2009) Association of major dietary patterns with socioeconomic and lifestyle factors of adult women living in Tehran, Iran. Nutrition 26, 337-341.

22. Willett W (1998) Nutritional Epidemiology. New York: Oxford University Press.

23. Kim J \& Mueller C (1978) Factor Analysis: Statistical Methods and Practical Issues. Beverly Hills, CA: Sage Publications, Inc.
24. Rimm E, Giovannucci E, Stampfer M, et al. (1992) Reproducibility and validity of an expanded self-administered semiquantitative food frequency questionnaire among male health professionals. Am J Epidemiol 135, 1114-1126.

25. Shu X, Yang G, Jin F, et al. (2004) Validity and reproducibility of the food frequency questionnaire used in the Shanghai Women's Health Study. Eur J Clin Nutr 58, 17-23.

26. Rosner B \& Willett W (1988) Interval estimates for correlation coefficients corrected for within-person variation: implications for study design and hypothesis testing. $A m \mathrm{~J}$ Epidemiol 127, 377-386.

27. Martin Bland J \& Altman D (1986) Statistical methods for assessing agreement between two methods of clinical measurement. Lancet 327, 307-310.

28. Ambrosini GL, O'Sullivan TA, de Klerk NH, et al. (2011) Relative validity of adolescent dietary patterns: a comparison of a FFQ and $3 \mathrm{~d}$ food record. Br J Nutr 105, 625-633.

29. Harika RK, Cosgrove MC, Osendarp SJM, et al. (2011) Fatty acid intakes of children and adolescents are not in line with the dietary intake recommendations for future cardiovascular health: a systematic review of dietary intake data from thirty countries. Br J Nutr 106, 307-316.

30. Hooper L, Summerbell CD, Higgins JP, et al. (2001) Reduced or modified dietary fat for preventing cardiovascular disease. The Cochrane Database of Systematic Reviews 2001 issue 3, CD002137.

31. Slattery M, Boucher K, Caan B, et al. (1998) Eating patterns and risk of colon cancer. Am J Epidemiol 148, 4-16.

32. Sánchez-Villegas A, Delgado-Rodríguez M, MartínezGonzález M, et al. (2003) Gender, age, socio-demographic and lifestyle factors associated with major dietary patterns in the Spanish Project SUN (Seguimiento Universidad de Navarra). Eur J Clin Nutr 57, 285-292.

33. Northstone K \& Emmett PM (2008) Are dietary patterns stable throughout early and mid-childhood? A birth cohort study. Br J Nutr 100, 1069-1076.

34. Martínez M, Marshall J \& Sechrest L (1998) Invited Commentary: factor analysis and the search for objectivity. $\mathrm{Am} \mathrm{J}$ Epidemiol 148, 17-19. 